\title{
Snapshot Hyperspectral Imaging (SHI) for Revealing Irreversible and Heterogeneous Plasmonic Processes
}

Silke R. Kirchner, ${ }^{\dagger} \|$ Kyle W. Smith, ${ }^{\dagger}\|\|$ Benjamin S. Hoener, ${ }^{\dagger}$ Sean S. E. Collins, ${ }^{\dagger}$ Wenxiao Wang, ${ }^{\dagger}$

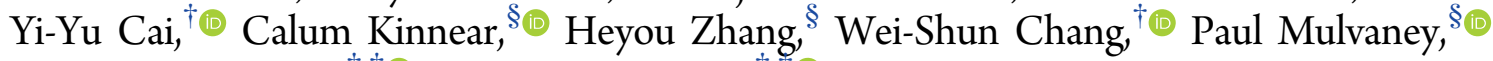

Christy F. Landes, $*,+, \neq$ and Stephan Link $*,+, *$ (i)

${ }^{\dagger}$ Department of Chemistry and ${ }^{\ddagger}$ Department of Electrical and Computer Engineering, Laboratory for Nanophotonics, Smalley-Curl Institute, Rice University, 6100 Main Street, Houston, Texas 77005, United States

${ }^{\S}$ School of Chemistry and Bio21 Institute, The University of Melbourne, Parkville, Victoria 3010, Australia

Supporting Information

ABSTRACT: Plasmon-mediated processes provide unique opportunities for selective photocatalysis, photovoltaics, and electrochemistry. Determining the influence of particle heterogeneity is an unsolved problem because often such processes introduce irreversible changes to the nanocatalysts and/or their surroundings. The challenge lies in monitoring heterogeneous nonequilibrium dynamics via the slow, serial methods that are intrinsic to almost all spectral acquisition methods with suitable spatial and/or spectral resolution. Here, we present a new metrology, snapshot hyperspectral imaging ( $\mathrm{SHI}$ ), that facilitates in situ readout of the tube lens image and first-order diffraction image of the dark-field scattering from many individual plasmonic nanoparticles to extract their respective spectra simultaneously. Evanescent wave excitation with a supercontinuum laser enabled signal-to-noise ratios greater than 100 with a time resolution of only 1 ms. Throughput of $\sim 100$ simultaneous spectra was achieved with a highly ordered nanoparticle array, yielding a spectral resolution of $0.21 \mathrm{~nm} /$ pixel. Additionally, an alternative dark-field excitation geometry utilized a combination of a supercontinuum laser and a reflecting objective for polarizationcontrolled SHI. Using a simplified version of SHI, we temporally resolve on the millisecond time scale the heterogeneous kinetics of an electrochemical surface redox reaction for many individual gold nanoparticles simultaneously.

\section{INTRODUCTION}

Plasmonic nanoparticles (NPs), characterized by their strong interaction with light and their high surface-to-volume ratio, play a crucial role as light-harvesting nanoantennas in photocatalysis, ${ }^{1-12}$ photovoltaics, ${ }^{13-15}$ plasmonic sensing, ${ }^{16-19}$ plasmon-mediated NP synthesis, ${ }^{20-28}$ surface-enhanced Raman spectroscopy, ${ }^{29-32}$ plasmonic tweezers, ${ }^{33}$ and spectroelectrochemistry. ${ }^{34-41}$ The photophysical response of a plasmonic nanoantenna strongly depends on its shape, ${ }^{28,42-51}$ surface morphology, ${ }^{52,53}$ material composition, ${ }^{54-57}$ and its environment and can furthermore be influenced by charge carrier density variations. ${ }^{58}$ During some applications, changes to the nanoantenna geometry can occur due to chemical reactions that take place at the surface. ${ }^{37,40}$ Single-particle spectroscopy has contributed tremendously to our understanding of the relationship between the size and shape of NPs and their plasmonic resonances. ${ }^{40,47,59,60}$ Conventional spectral imaging techniques are often limited by a combination of real-time performance for a single (or few) nanostructure(s), sensitivity (i.e., spectral resolution), and NP throughput. Consequentially, there is a dearth of knowledge regarding the way NP polydispersity can affect irreversible and fast plasmon-mediated processes. Understanding the photophysical and photochemical effects of sample heterogeneity on plasmonically triggered reactions is an important first step toward characterizing hotcarrier-induced surface chemistry and catalysis as well as effective tailoring of nanostructure geometry for successful applications.

Assuming a constant rate of optical excitation, spectral detection techniques can be assessed using the following parameters: the number of NPs measured at a time, $N_{\mathrm{NP}}\left(t_{0}\right)$, the required acquisition time of the detector, $\delta t_{\mathrm{aq}}$, the spectral resolution, $R\left(\delta t_{\mathrm{aq}}\right)$, and the signal-to-noise ratio $^{61-63}$ in the recorded wavelength spectrum $\left(\mathrm{S} / \mathrm{N}\left(\delta t_{\mathrm{aq}}\right)\right)$. Several experimental methods have been developed to reveal the optical characteristics of plasmonic NPs, but the ideal combination of all four detection parameters has not been reported yet. In UV-vis-NIR spectroscopy, the spatial throughput of NPs

\footnotetext{
Received: February 8, 2018

Revised: February 28, 2018

Published: March 1, 2018
} 
exceeds the maximum number of particles allowed to extract single-particle spectra and $N_{\mathrm{NP}}\left(t_{0}\right) \gg N_{\text {ideal }}\left(t_{0}\right)$. Although current single-particle spectroscopy can achieve fast camera integration times down to tens of microseconds, ${ }^{64} \delta t_{\text {aq. }} \cong \delta t_{\text {ideal }}$, if the incident photon flux is high, it is restricted to the investigation of a single (or few) $\mathrm{NP}(\mathrm{s})$ at a time, $N_{\mathrm{NP}}\left(t_{0}\right) \ll$ $N_{\text {ideal }}\left(t_{0}\right)$. In contrast, spatial hyperspectral imaging ${ }^{40}$ by scanning the sample or the wavelength enables the measurement of many individual plasmonic NPs, $N_{\mathrm{NP}}\left(t_{0}\right) \cong N_{\text {ideal }}\left(t_{0}\right)$, but the overall measurement time depends on the number of measured NPs, $\delta t_{\text {aq. }} \gg \delta t_{\text {ideal }}{ }^{65,66}$ or the spectral resolution is low, $\left.R\left(\delta t_{\text {aq. }}\right) \ll R_{\text {ideal }}\left(\delta t_{\text {aq. }}\right)\right)^{66}$ Spectral resolutions better than the limits determined by the hardware can in principle be achieved by fitting single-particle spectra as demonstrated in single-particle plasmon resonance sensing experiments that illustrated analyte detection with an accuracy of $0.03 \mathrm{~nm}$ at a 10 ms detector exposure time. ${ }^{17,18}$ Finally, transmission gratingbased spectroscopic approaches that have been described so far require detector acquisition times from 35 to over $100 \mathrm{~ms}\left(\delta t_{\text {aq }}\right.$. $\left.\gg \delta t_{\text {ideal }}\right)$ and/or the spectral resolution is low, $R\left(\delta t_{\text {aq. }}\right) \stackrel{\text { }}{\ll}$ $R_{\text {ideal }}\left(\delta t_{\text {aq. }}\right)$, varying between 1.6 and $7 \mathrm{~nm}$ per pixel. ${ }^{67-69}$ Such low spectral sensitivity hinders a precise measurement of wavelength shifts on the single nanometer scale that can occur, for example, in spectroelectrochemical reactions even if spectra were fitted to Lorenztian curves. ${ }^{39}$ In addition, spatial throughput is often not optimized as the zeroth and first orders of diffraction are detected with the same camera and thus $N_{\mathrm{NP}}\left(t_{0}\right) \ll N_{\text {ideal }}\left(t_{0}\right)$. $^{68-73}$

In this work, we present a technique that provides in situ spectroscopic mapping of many individual nanostructures in a fast and truly parallel manner, rendering discernible signals on a millisecond time scale with high signal-to-noise $(\mathrm{S} / \mathrm{N})$ ratio. ${ }^{61-63}$ This advancement is possible through the optimization of all four detection parameters at the same time using a novel snapshot hyperspectral imaging ( $\mathrm{SHI}$ ) methodology. The key features of the SHI setup are a diffracting optical element (a transmission grating) and two complementary metal-oxide-semiconductor (CMOS) cameras. The two cameras enable separate and parallel readout of the spatial and spectral dark-field scattering images of multiple single NPs on a millisecond time scale. The precise correlation of the two CMOS cameras facilitates the extraction of the respective NP wavelength spectra, which exhibit $\mathrm{S} / \mathrm{N}$ ratios up to $\left(\mathrm{S} / \mathrm{N}\left(\delta t_{\mathrm{aq}}\right)\right) 125$ for $\delta t_{\mathrm{aq} .}=1 \mathrm{~ms}$, whereas the spectral resolution is set to $R(1 \mathrm{~ms})=0.21 \mathrm{~nm} /$ pixel. Our experimentally determined NP throughput is $N_{\mathrm{NP}}\left(t_{0}\right) \cong 100$ at a time for ordered NP arrays. For randomly positioned NPs, overlapping spectra reduce this number to about 20, comparable to closely related prior work that demonstrated parallel spectroscopic imaging by inserting a spatially addressable liquid crystal filter in the detection path. ${ }^{74}$ Finally, we present a novel dark-field excitation approach that utilizes a combination of a supercontinuum laser and a reflecting objective for polarization-controlled SHI.

\section{RESULTS AND DISCUSSION}

The principle of our detection system is based on the synchronized acquisition of the spatial positions and the spectral dispersions of many single NPs. Our SHI setup comprised a beam splitter, a transmission grating, and two CMOS cameras (ORCA-flash 4.0 V2, Hamamatsu, Figure 1a). We used the first CMOS camera (CMOS 1) to detect the spatial tube lens image and the second CMOS camera (CMOS (a)

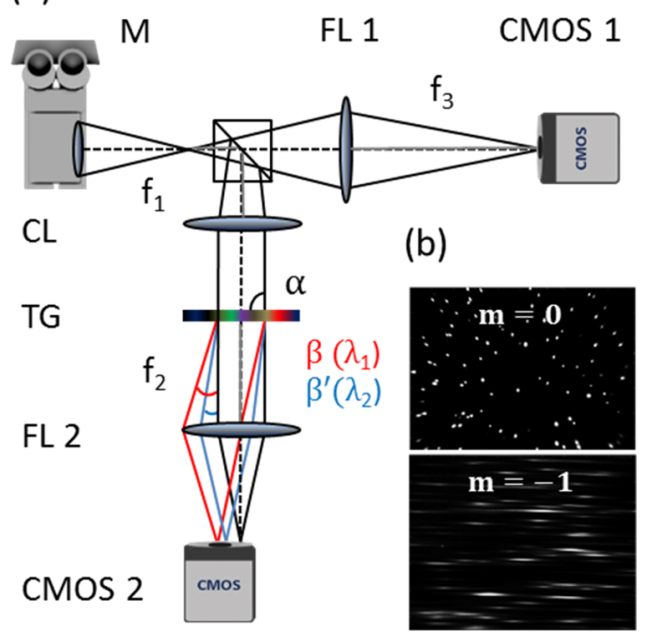

Figure 1. Snapshot hyperspectral imaging. (a) The image of the microscope $(\mathrm{M})$ tube lens is split into two parts by a beam splitter. The transmitted spatial image is focused (FL 1) on the first CMOS camera (CMOS 1). The reflected image is collimated (CL) and diffracted by a transmission grating (TG) positioned normally to the light propagation direction $\left(\alpha=90^{\circ}\right)$. The generated spectral image is refocused (FL 2) on the second CMOS camera (CMOS 2). (b) Spatial $(m=0)$ and spectral $(m=-1)$ dark-field scattering images of multiple single AuNRs simultaneously detected by CMOS 1 and CMOS 2, respectively.

2) to simultaneously reveal the spectral dispersion of the image after it passed the transmission grating (Figure 1a).

The image of the tube lens of an inverted microscope (Axiovert, Zeiss) was split with a nonpolarizing beam splitter cube having a 70:30 reflectance-transmittance ratio (Thorlabs). The transmitted image was then focused on the CMOS 1 camera by an achromatic doublet lens (Newport). The lens had an effective focal length of $f_{3}=80 \mathrm{~mm}$ and was positioned to achieve a $4 f$ geometry keeping the magnification of the microscope at the CMOS 1 camera the same. This one-toone image projection ensured minimal photon loss while the complete field of view of the objective was projected on the active pixel sensor of the CMOS 1 camera.

For the spectral detection of the NPs, the reflected and hence inverted portion of the tube lens image was collimated by an achromatic doublet lens having an effective focal length of $f_{1}=$ $100 \mathrm{~mm}$ (Figure 1a). The collimated light passed a transmission grating (Edmund Optics) with a groove density of 300 grooves $/ \mathrm{mm}$ and a blaze angle of $17.5^{\circ}$. The second CMOS camera (CMOS 2) was positioned $200 \mathrm{~mm}$ behind the transmission grating. A visible achromatic doublet lens with an effective focal length of $f_{2}=100 \mathrm{~mm}$ (Newport) was placed in front of the CMOS 2 camera to focus the transmitted firstorder diffraction of the image on the camera chip (Figure 1a). As the transmission grating was normally aligned to the photon propagation direction $\left(\alpha=90^{\circ}\right.$, Figure 1a), the transmission grating equation reads as

$$
\beta\left(m, \lambda_{n}\right)=\arcsin (m \lambda / \Delta)
$$

with diffraction angle $\beta$, wavelength of light $\lambda_{n}$, order of diffraction $m$, and grating period $\Delta$. From this equation, one can see that the diffraction angle depends only on the order of diffraction as well as the wavelength for a constant grating period; the angle of diffraction increases with the wavelength 
(a)

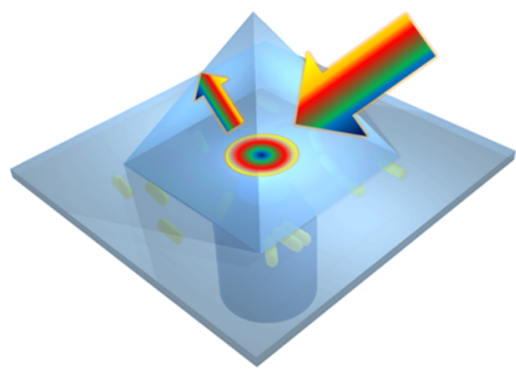

(b)

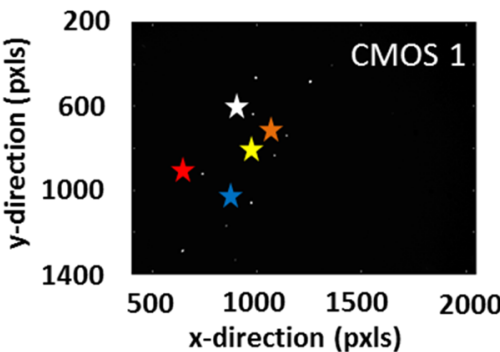

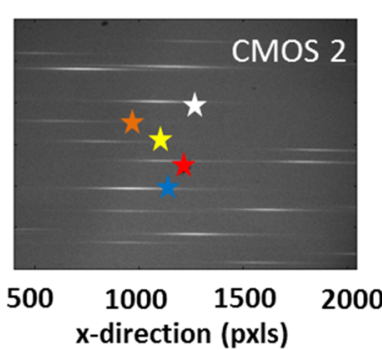

(c)

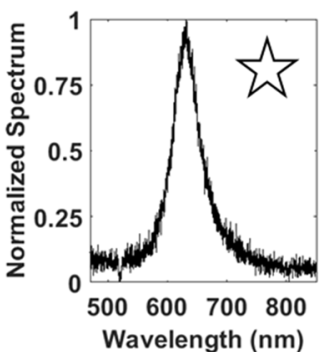

Figure 2. Halogen lamp prism-TIRS excitation of AuNRs and SHI detection. (a) By means of a halogen lamp and a prism, TIR conditions were generated at the substrate-air interface. AuNRs $(41 \mathrm{~nm} \times 60 \mathrm{~nm})$ deposited on the back side of the cover slip were excited by the evanescent wave, and the resonantly scattered light was collected with a $50 \times$ air-spaced objective. (b) Spatial and spectral scattering images of the AuNRs were simultaneously detected by CMOS 1 and CMOS 2 cameras. The colored stars correlate example AuNR positions on the CMOS 1 camera with the respective dispersions (i.e., spectra) on the CMOS 2 camera. (c) Extracted spectrum of an individual AuNR. The comparison of the spectra collected for the same AuNR using SHI and a conventional spectrograph together with a CCD camera showed very good agreement (Supporting Information Section 4, Figure S7).

and decreases with a larger grating period. In general, the spectral range that can be collected with the CMOS 2 camera depends on the grating groove density, the magnification provided by the collimating and focusing lenses, and the sensor size of the camera. The active area of the CMOS camera had a size of $13.312 \times 13.312 \mathrm{~mm}^{2}$ or $2048 \times 2048$ pixel $^{2}$. The sensor size together with the set of lenses used provided a measureable spectral dispersion from $\lambda_{\min }=430 \mathrm{~nm}$ to $\lambda_{\max }=870 \mathrm{~nm}$ when the NP was positioned in the center of the CMOS 1 camera (Supporting Information 1, Figure S1, Table S1). This corresponded to a spectral resolution of $0.21 \mathrm{~nm} /$ pixel for our SHI setup. (The calibration details for the CMOS 1 and CMOS 2 cameras are described in the Supporting Information Section 1, Figures S1-S3.)

The theoretical spectral sensitivity of our technique is of the same order as that of the optical resolution provided by common spectroscopic detection methods. In comparison, a slit spectrograph combined with a CCD camera (e.g., SP-2150i and PIXIS/PyLoN:100/400 from Princeton Instruments) provides a spectral resolution of $0.38 \mathrm{~nm} /$ pixel for a slit width of $20 \mu \mathrm{m}$ and a grating with 300 grooves $/ \mathrm{mm}$. The combination of the chosen transmission grating together with the particular set of lenses provided the most appropriate balance between photon efficiency and spectral resolution. To visualize the spatial and spectral output of the SHI approach, we show the detection of individual gold nanorods (AuNRs) in Figure 1b. The CMOS 1 camera provides the spatial image of the AuNRs, whereas the CMOS 2 camera simultaneously measures the spectral dispersion of each AuNR. By utilizing two cameras for the separate detection of the tube lens and first-order diffraction images, we extended the possible simultaneous spatial NP throughput without any loss of spectral sensitivity, in comparison with previous work utilizing only one CMOS detector. $^{67,68,73}$
As our SHI methodology does not involve an entrance slit, a dark-field excitation configuration of the investigated NPs is necessary for their distinct detection. We used an evanescent wave to optically excite the resonant scattering of plasmonic NPs and successfully extracted their respective spectra with respect to an otherwise dark background. For the evanescent wave excitation, a prism was brought in contact with the back side of a cover slip (Figure 2a).

Index matching was achieved with microscope objective immersion oil. The light of a $250 \mathrm{~W}$ halogen lamp (Newport) was directed through the prism and focused at the cover slip by a lens $(f=35 \mathrm{~mm})$ under the critical angle for total internal reflection (TIR). The evanescent wave generated at the cover slip-air interface excited the AuNRs (see Supporting Information Section 2.1 for detailed AuNR characterization). The resonantly scattered light of the AuNRs was collected by a $50 \times$ air-spaced objective (Zeiss). The image was additionally magnified by a factor of 2.5 by an internal lens of the microscope, leading to an effective magnification of 125 . The exposure time of the CMOS 1 and CMOS 2 cameras was set to $1 \mathrm{~s}$, and the tube lens and first-order diffraction images were captured (Figure 2b). The positions of the single NPs recorded by the CMOS 1 camera were correlated to the respective spectral dispersions captured by the CMOS 2 camera (colored stars in Figure 2b). The full width of the spectral dispersion of an individual AuNR on the CMOS 2 camera chip was found to be $\sim 400$ pixel (i.e., $\sim 84 \mathrm{~nm}$ ) in the $x$-direction and $\sim 20$ pixel (i.e., $\sim 4 \mathrm{~nm}$ ) in the $y$-direction for our particular setup parameters. To extract wavelength-calibrated spectra for all AuNRs (Figure 2c), we converted the position-dependent dispersions of each NP into wavelength information using a linear function (see Supporting Information Sections 1 and 3.1 for further details). The excitation power spectrum (Figures S4 and S5) was largely uniform across the spectral region of 
interest, making a normalization for AuNR spectra not necessary. We furthermore verified the correctness of this procedure by performing reference measurements with a regular spectrograph together with a CCD camera (Princeton Instruments, Acton SpectraPro 2150i). We measured the spectra of the same AuNRs with both approaches, and the agreement was very good (see Supporting Information Section 4, Figure S7).

Besides the high spatial throughput of NPs and spectral resolution, a large signal-to-noise ratio, $\mathrm{S} / \mathrm{N}\left(\delta t_{\mathrm{aq}}\right)$, is important for the acquisition of many high-quality spectra. We achieved high $\mathrm{S} / \mathrm{N}$ ratios for CMOS camera acquisition times of only 1 $\mathrm{ms}$ by utilizing a supercontinuum laser for the evanescent wave excitation. The photon flux that reaches the photodetectors is a crucial parameter for the acquisition-time-dependent $\mathrm{S} / \mathrm{N}$ ratio of a NP spectrum. To considerably enhance the scattering intensity of individual AuNRs, we therefore exchanged the halogen lamp in the prism-TIR configuration by a high-power white light fiber laser (WLL, WhiteLase SC480, Fianium) with a total output power of $10 \mathrm{~W}$ over a wavelength range of 480$2400 \mathrm{~nm}$ (Figure 3a).

To avoid sample heating and match the excitation wavelength to the detection window, wavelengths greater than 900 $\mathrm{nm}$ were reflected by a dichroic mirror (Chroma) after the laser output. The beam was then expanded three times by a Keplerian telescope constructed from two plano-convex, achromatic lenses with focal lengths of $f_{1}=50 \mathrm{~mm}$ and $f_{2}=$ $150 \mathrm{~mm}$ (Newport) placed at a distance of $200 \mathrm{~mm}$ from each other. The WLL was focused by an achromatic lens $(f=88$ $\mathrm{mm}$, Newport) on the back side of the cover slip that was mounted on the microscope stage. The propagation direction of the beam matched the critical angle for TIR (Figure 3a), and the generated evanescent wave of the WLL prism-TIR excitation configuration led to high-intensity scattering of the AuNRs over a dark background (Figure 3b, see Supporting Information Section 2.2, Figure S6 for the AuNR synthesis and characterization). The resonantly scattered light was collected by the same $50 \times$ objective, and the image was again magnified by a factor of 2.5 using the internal microscope lens. The exposure time of the CMOS 1 and CMOS 2 cameras was set to $1 \mathrm{~ms}$ (vs $1 \mathrm{~s}$ previously), and the tube lens and first-order diffraction images were captured (Figure 3c). The spatial image of each single AuNR was correlated to its spectral image (white stars in Figure 3c), and the spectra were extracted following the wavelength calibration and correction procedure described in the Supporting Information. The WLL prism-TIR excitation of the AuNRs resulted in NP spectra that showed high $\mathrm{S} / \mathrm{N}$ ratios, while using only a $1 \mathrm{~ms}$ acquisition time for both CMOS cameras (Figure 3d, left) and a total output laser power of 10 W. A detailed analysis of the acquisition-time-dependent $\mathrm{S} / \mathrm{N}$ ratio will be discussed below. In some regions of the spectral map, superposition of dispersions from several AuNRs occurred (red stars in Figure 3c). As a consequence, the corresponding spectra showed features of two or more AuNRs (Figure 3d, right). On average, we extracted 10-20 simultaneous spectra from randomly deposited AuNRs with a spectral resolution of $0.21 \mathrm{~nm} /$ pixel, comparable to that in previous pioneering work $^{74}$ that used a liquid crystal shutter to avoid the issue of overlapping spectra.

To estimate the spatial throughput of the SHI setup while excluding superposition of dispersions, we measured an ordered array of individual plasmonic AuNRs. We found the experimental throughput of the SHI approach to be $N_{\mathrm{NP}}\left(t_{0}\right)$ (a)

(b)
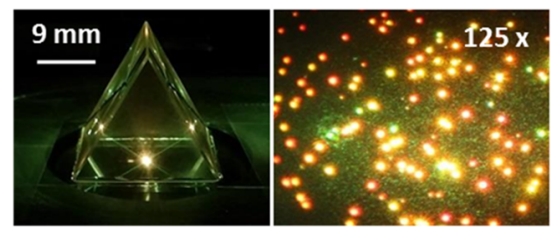

(c)
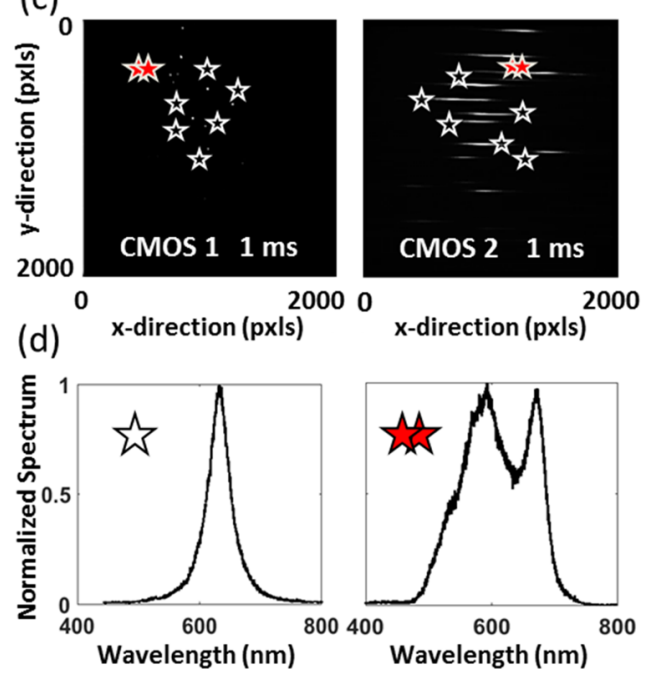

Figure 3. WLL prism-TIRS excitation (WLL/PRISM) of AuNRs enables a $1 \mathrm{~ms}$ camera exposure time. (a) WLL and a prism were used to establish TIR conditions on the substrate-air interface of a cover slip. The bright spot in the prism center is the focus of the WLL where TIR is generated. (b) SLR camera image of the high-intensity scattering of single AuNRs (32 nm $\times 78 \mathrm{~nm}$, Figure S6) deposited on the back side of the cover slip. (c) Spatial and spectral maps of the scattering of multiple individual AuNRs recorded by CMOS 1 and CMOS 2 cameras within $1 \mathrm{~ms}$. The white stars correlate the AuNR positions to their respective spectral dispersions. Spectra (red stars) overlapped when the interparticle distance was less than $\sim 400$ pixel $(\sim 21 \mu \mathrm{m})$ in the $x$-direction and $\sim 20$ pixel $(\sim 1 \mu \mathrm{m})$ in the $y$-direction for a $125 \times$ magnification. (d) Single AuNR spectrum reveals a high $S$ / $\mathrm{N}$ ratio for a camera acquisition time of only $1 \mathrm{~ms}$ (left). The extracted spectral information for overlapping dispersions is shown in the right panel.

$\cong 100$ NPs under our conditions, i.e., for a $125 \times$ magnification and a corresponding full spectral width of a single AuNR on the CMOS 2 camera of $\sim 400$ pixel (i.e., $\sim 84 \mathrm{~nm}$ ) in the $x$-direction and $\sim 20$ pixel (i.e., $\sim 1 \mu \mathrm{m}$ in spatial dimensions) in the $y$ direction. In the ideal case of no spectral superposition and a sensor area composed of $2048 \times 2048$ pixel $^{2}$, up to 500 individual AuNRs could theoretically be detected with the CMOS 2 camera. Although this number represents an upper limit, we tested the NP throughput capabilities of SHI experimentally with an AuNR sample that was fabricated by electron-beam lithography (please see Supporting Information Section 10 for methodical details). The AuNRs had dimensions of $40 \mathrm{~nm} \times 80 \mathrm{~nm}$ and were supported on a quartz substrate. As the camera field of view was $106 \times 106 \mu \mathrm{m}^{2}$ (i.e., $2048 \times$ 2048 pixel $^{2}$ ) for the used magnification, we chose an interparticle distance of $60 \mu \mathrm{m}$ (i.e., $\sim 1159$ pixel) in the $x$ direction and a distance of $1.5 \mu \mathrm{m}$ (i.e., $\sim 29$ pixel) in the $y$ direction to avoid spectral superposition at the CMOS 2 camera (Figure 4a).

The AuNRs were excited with the WLL prism-TIR approach. Figure $4 \mathrm{~b}$ depicts the spectroscopic recording of the AuNR 
(a)

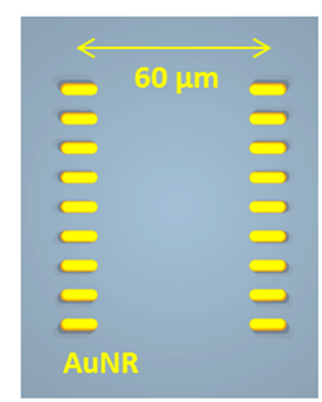

(c)
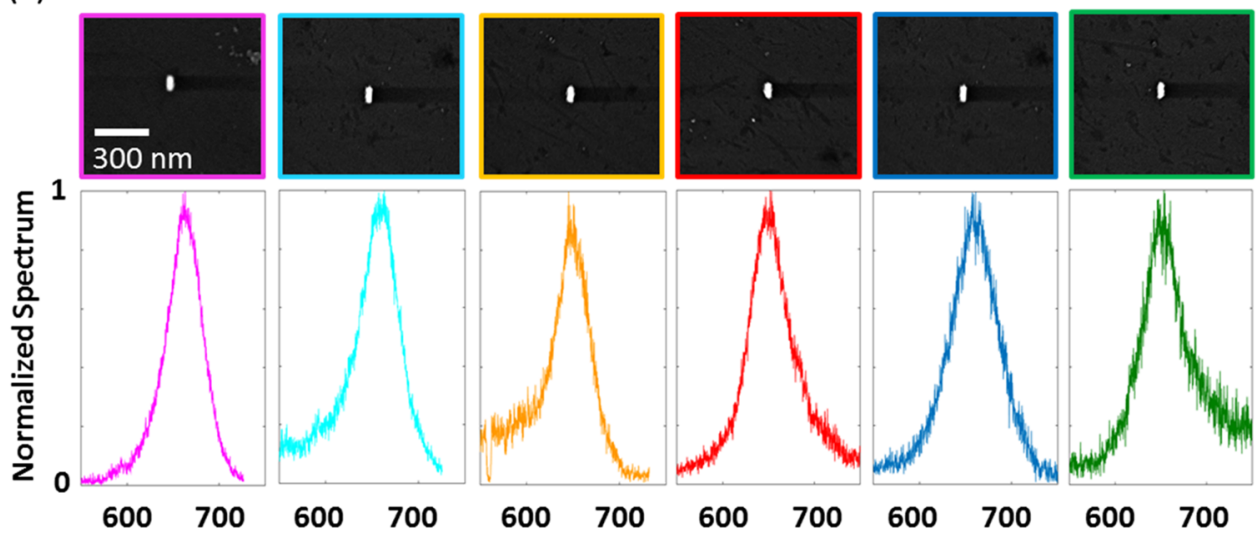

600700

(b)

CMOS 1

CMOS 2
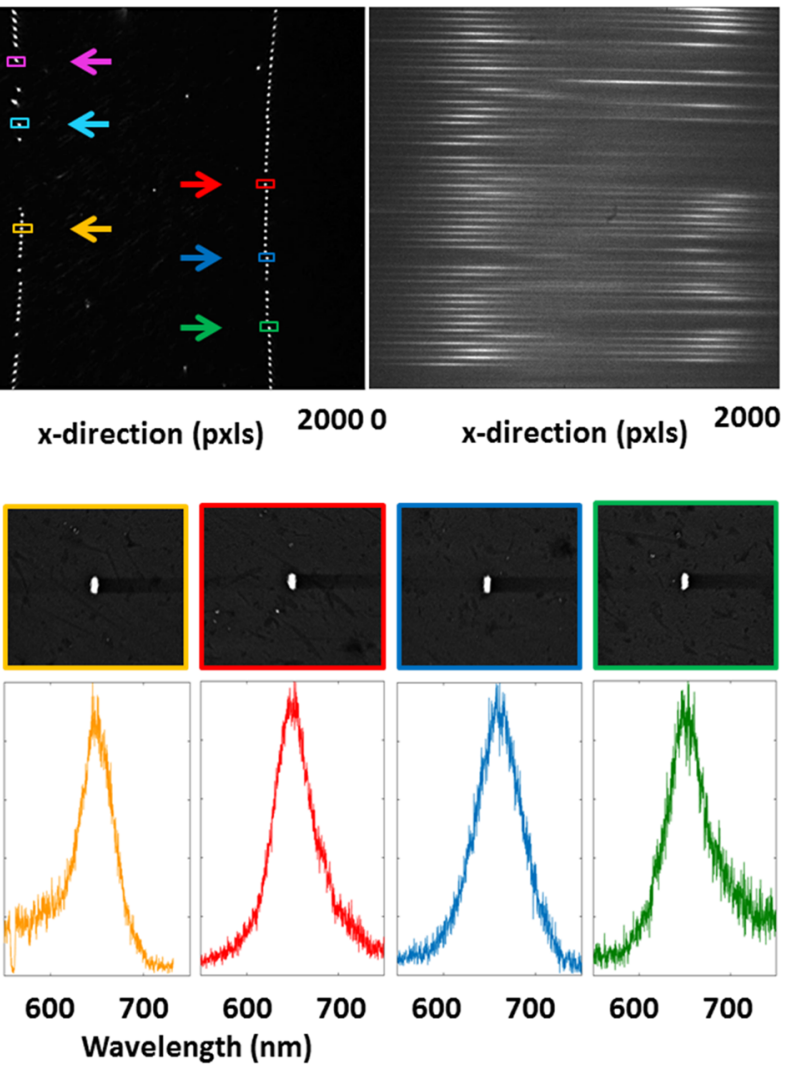

Figure 4. Measurement of 83 AuNR spectra within $1 \mathrm{~ms}$. AuNRs (40 nm $\times 80 \mathrm{~nm}$ ) were equidistantly positioned on a quartz substrate using e-beam lithography. (a) NPs had an interparticle distance of $60 \mu \mathrm{m}(\sim 1159$ pixel) in the $x$-direction and $1.5 \mu \mathrm{m}$ ( $\sim 29$ pixel) in the $y$-direction. (b) CMOS 1 (left) and CMOS 2 (right) camera images of the AuNR array taken with an integration time of $1 \mathrm{~ms}$. A total of $53 \mathrm{NPs}$ fit into the field of view of the CMOS cameras in the $y$-direction, and two rows were imaged in the $x$-direction for this particular arrangement of sample and optical setup with a $0.21 \mathrm{~nm} /$ pixel wavelength resolution. In the case of a perfect AuNR array (a few AuNRs did not develop), more than 100 NPs can be simultaneously detected within $1 \mathrm{~ms}$ under these conditions. (c) Examples of extracted spectra. The colors of the spectra match the colors of the arrows in (b).

array with the two CMOS cameras. In this experiment, the spectral information of 83 AuNRs was acquired within only 1 ms. Several examples of extracted spectra are shown in Figure 4c. A total of $53 \mathrm{NPs}$ fit into the field of view of the CMOS cameras in the $y$-direction (Figure $4 \mathrm{~b}$, left image). With two parallel columns, no overlap of individual dispersions occurred in either direction (Figure 4b, right image). As some AuNRs were missing, the number of recorded spectra was slightly lower than that for a perfect AuNR pattern. In addition, we cropped the edges of the captured images in the $x$-direction (Figure $4 \mathrm{~b}$ ) because of coma effects in the optical system. A correction for the coma aberration, e.g., via deconvolution of a blurred Airy disk image, would enable a full image readout and then the in situ detection of 140 individual AuNRs within $1 \mathrm{~ms}$.

To make SHI detection more cost-effective and more accessible to other groups, we have implemented a single CMOS detector version. ${ }^{67,68,71-73,75}$ In this single detector setup, we exchanged the original transmission grating (300 l/ $\mathrm{mm}$ ) with a coarser transmission grating having a line density of $70 \mathrm{l} / \mathrm{mm}$. We therefore sacrificed some spectral resolution $(0.85 \mathrm{~nm} /$ pixel for the $70 \mathrm{l} / \mathrm{mm}$ grating instead of $0.21 \mathrm{~nm} /$ pixel for the $300 \mathrm{l} / \mathrm{mm}$ grating). However, the positive trade-off is in cost as only one CMOS detector is required. Additionally, alignment and calibration are simplified. Utilizing this single detector setup, we established the unique application possibilities of SHI. We temporally resolved the millisecond kinetics of an electrochemical redox reaction of many single AuNRs in parallel under the same conditions by monitoring the changes of their LSPR wavelengths and thereby observing diverse optical changes in response to a reversible formation of gold oxide layers on the particle surfaces (Figure 5, experimental conditions are described in Section 8 of the Supporting Information).

As shown in Figure 5a,b, at a positive electrochemical potential of $+1.0 \mathrm{~V}$, the scattering spectra of multiple single AuNRs, measured at the exactly same time, red-shifted and were strongly damped because of capacitive charging and oxidation of the $\mathrm{Au}$ surface. As soon as the potential was switched back to $-0.2 \mathrm{~V}$, the AuNR spectra reversibly recovered to their original values. For AuNRs in a $100 \mathrm{mM}$ $\mathrm{NaF}$ electrolyte solution, the electrochemical reaction that occurs at the lowest positive potential is adsorption of hydroxide and oxidation of the gold surface. The formation of $\mathrm{Au}_{x} \mathrm{O}_{y}$ changes the local refractive index around the AuNR and leads to a red shift of the plasmon resonance. Chemical interface damping likely also contributed as the plasmon resonance intensity decreased and the line width broadened. Although this behavior is consistent with the results from several groups that reported electrochemically produced $\mathrm{Au}_{x} \mathrm{O}_{y}$ on a gold surface at a potential of $1.5-1.7 \mathrm{~V}$ relative to a standard hydrogen electrode (SHE) and estimated a $\mathrm{Au}_{x} \mathrm{O}_{y}$ thickness of $0.4-1.1 \mathrm{~nm},{ }^{76-79}$ no time-resolved studies have yet 
(a)

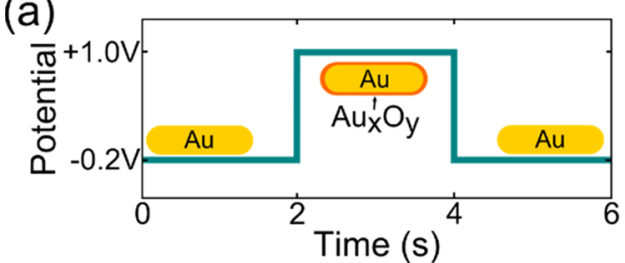

(b)

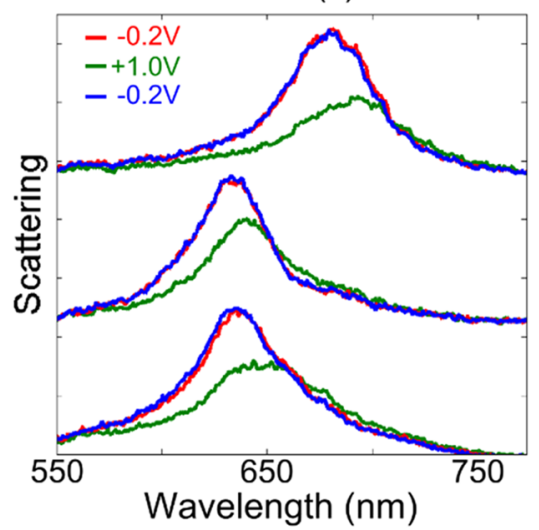

(c)

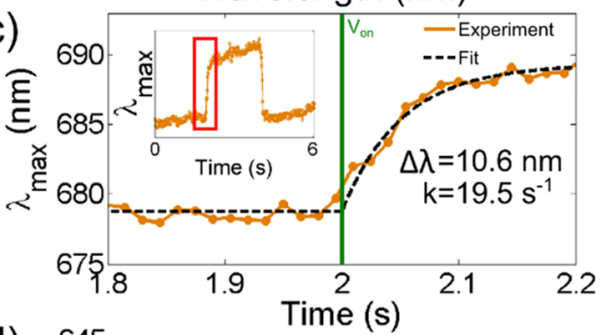

(d)

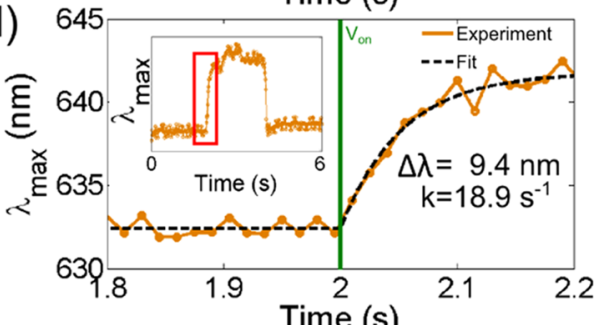

(e)

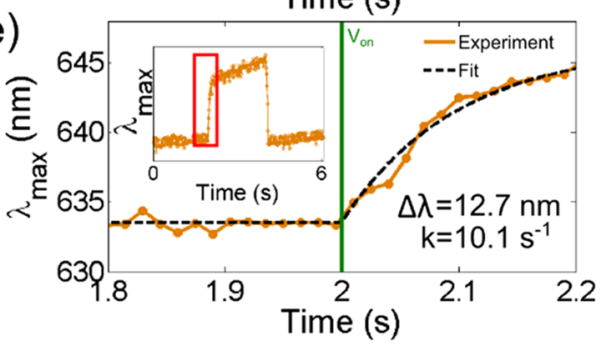

Figure 5. Temporally resolved spectral shifts from individual AuNRs subjected to an electrochemical redox reaction, measured with millisecond time resolution in parallel, to demonstrate the capabilities of SHI. (a) Applied potential was varied from -0.2 to $+1.0 \mathrm{~V}$ in a step function with a $2 \mathrm{~s}$ time period. (b) Selected scattering spectra of three AuNRs illustrating reversible LSPR shifts. At positive potentials $(+1.0$ V), the scattering spectra of multiple single AuNRs red-shifted and broadened because of capacitive charging and oxidation of the $\mathrm{Au}$ surface. When the potential was switched back to $-0.2 \mathrm{~V}$, the AuNR spectra recovered to the original position because of oxide reduction. (c-e) Temporally resolved peak LSPR wavelengths of the same three AuNRs as in (b) in response to the applied potential switch, acquired with a time resolution of $20 \mathrm{~ms}$. The dashed black line is a fit to eq 2 (see the text for details). The inset shows the time evolution over a longer time period demonstrating both oxidation and reduction. been performed. We therefore closely investigated the temporal development of the LSPR shifts upon gold oxide formation. The maximum LSPR shifts were reached at about $200 \mathrm{~ms}$ after applying the electrochemical potential step (Figure 5a). As examples, insets of Figure $5 \mathrm{c}-\mathrm{e}$ show the temporal evolution of the resonance maxima of the same three individual AuNRs as in part $b$, measured simultaneously under the same conditions as a function of time with a time resolution of $20 \mathrm{~ms}$. The average red shift at $1.0 \mathrm{~V}$ (corresponding to $1.5 \mathrm{~V}$ relative to SHE) for 17 AuNRs was $12.1 \mathrm{~nm}$. By expanding the time traces (main parts of Figure $5 c-e$ ), we were able to fit the time-dependent wavelength shift to an exponential rise according to the following equation

$$
\lambda_{\max }=\Delta \lambda\left(1-\mathrm{e}^{-k(t-2 s)}\right)+\lambda_{i}
$$

where $\Delta \lambda$ and $k$ are fit parameters. The initial resonance wavelength, $\lambda_{\mathrm{i}}$, was taken as the average resonance position for the first 50 exposures, and $2 \mathrm{~s}$ corresponds to the time at which the applied voltage was changed.

The values of $k$ obtained this way ranged from 10.1 to 23.2 $\mathrm{s}^{-1}$, while the reduction was faster than our time resolution. This spread in reaction kinetics demonstrates the heterogeneity of a typical electrochemical redox reaction that must be monitored under the exactly same experimental conditions in parallel for many NPs. In addition to the heterogeneity in reaction kinetics, we observed some dramatic outliers for the measured LSPR wavelength shifts upon Au oxidation. In particular, one of the AuNRs showed a significantly smaller LSPR shift of less than $2 \mathrm{~nm}$ (Figure S14). On the basis of this data, even smaller wavelength shifts of $\lambda<1 \mathrm{~nm}$ should be achievable. The stability of our SHI setup furthermore supports this claim, as demonstrated by measuring the resonance wavelengths of 93 AuNRs in parallel. Over a measurement period of $6 \mathrm{~s}$, the wavelength stability was $0.57 \mathrm{~nm}$ with a standard deviation of $0.11 \mathrm{~nm}$ (Supporting Information Section 9, Figure S15).

As the spectral investigation of nonisotropic plasmonic NPs requires a precise control of the polarization of the excitation light, we present an innovative high-intensity dark-field approach that can be used with SHI. This method utilized a WLL with normal incidence onto the sample from above and a reflecting objective (WLL/RO) positioned below (Figure 6a).

A polarizing optical element was easily implemented before the sample. While evanescent wave excitation limits the degree of polarization control, ${ }^{80}$ the WLL/RO geometry represents a great alternative for exact, polarization-dependent measurements. It furthermore provides a way to vary the incident $k$ vector, important as samples are typically not rotated. In detail, we coupled the WLL into the microscope such that it was normally incident on an aluminum-coated, reflecting objective (74×/0.65, Beck Optronic Solutions). An achromatic lens in front of the objective with an effective focal length of $f=100$ $\mathrm{mm}$ (Newport) was used to focus the incoming beam to the center of the light stop of the objective. As a consequence, direct excitation light from the laser was blocked by the light stop, resulting in a dark background, as required for SHI. We positioned the sample substrate on a microscope stage with AuNRs facing toward the reflecting objective in between the focusing lens and the reflecting objective (see Supporting Information Section 2.3 for further details regarding the sample). When NPs were in focus of the reflecting objective, only light resonantly scattered by the individual AuNRs reached 
(a)

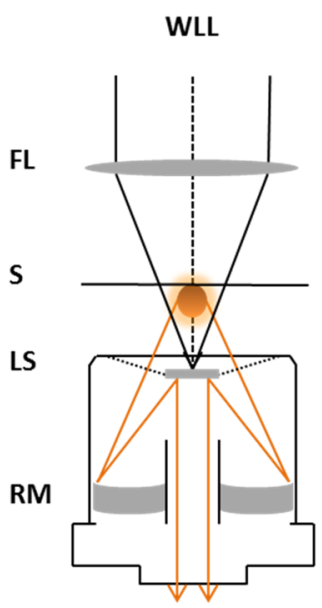

DF (b)

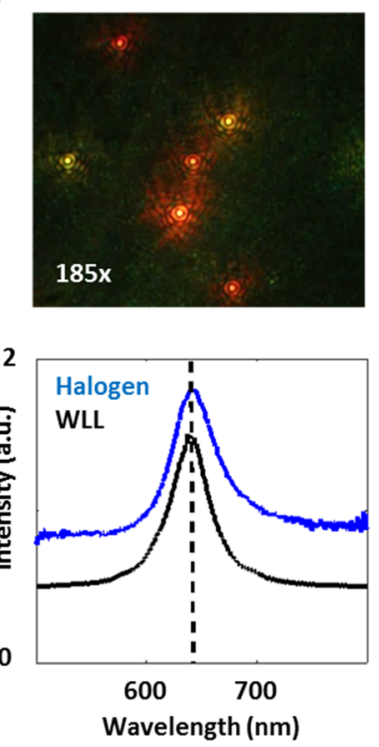

(d)

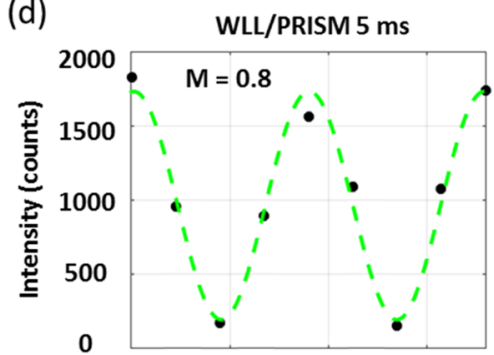

(e)

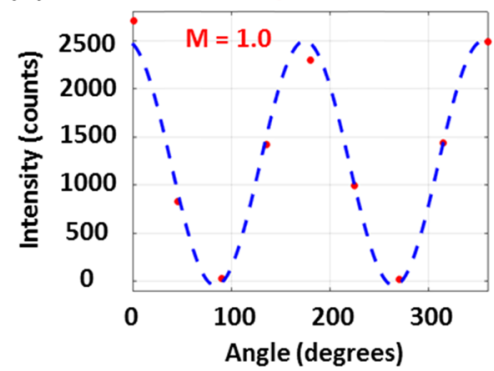

Figure 6. Dark-field (DF) detection using a WLL in combination with a reflecting objective (WLL/RO). (a) WLL was focused with an achromatic lens (FL) at normal incidence on the sample (S) and into an aluminum-coated reflecting objective at the center of its light stop (LS). Consequently, the zeroth-order diffraction of the laser beam (i.e., the directly transmitted beam) was blocked by the light stop and only light isotopically scattered by a NP was reflected by the objective mirrors (RM) and reached the detectors. (b) SLR camera image (185X magnification) of the high-intensity dark-field scattering of single AuNRs $(41 \mathrm{~nm} \times 60 \mathrm{~nm}$ ). (c) Comparison of the spectra measured with the WLL/RO method and a regular halogen dark-field illumination for the same AuNR showed good agreement. The latter measurement was taken with a conventional spectrograph and a CCD camera. In this case, the exposure time was $1 \mathrm{~s}$ for both excitation schemes. Polarization-angle-dependent intensity mapping of the scattering for two individual AuNRs excited with the WLL/PRISM (d) and the WLL/RO configuration (e). The WLL/RO measurement shows a greater modulation depth $M(1.0$ vs 0.8$)$ than the WLL/PRISM approach but requires longer CMOS 2 camera acquisition times (30 vs 5 ms) for similar integrated scattering intensities.

the back focal plane of the objective (Figure 6a,b). This configuration led to a high scattering intensity of the NPs on a dark background (Figure $6 \mathrm{~b}$ ) and allowed us to measure their spectra, first using a conventional spectrograph and CCD camera (Figure 6c, Supporting Information Section 3.2). To verify the accuracy of the measured AuNR spectra with the WLL/RO approach, we also recorded spectra of the same AuNRs using a regular halogen lamp (Zeiss) dark-field illumination. The agreement between these two methods is very good, as illustrated in Figure 6c.

To evaluate the performance of the WLL/RO illumination, we quantitatively compared the $\mathrm{S} / \mathrm{N}$ ratios of the wavelength spectra as well as the polarization-dependent scattering intensities of AuNRs for the WLL/RO and the WLL prismTIR (WLL/PRISM) geometries. We found that SHI measurements exhibited better polarization control with the WLL/RO method but yielded smaller $\mathrm{S} / \mathrm{N}$ ratios compared to those from the WLL/PRISM approach when using the same camera integration times. The signal recorded with a CMOS photodetector depends on the photon flux incident on the camera sensor, the quantum efficiency of the device, and the integration time over which the signal is collected, whereas the three primary contributors to the noise are the photon noise (from the Poisson distribution in arrival times of photons), dark noise (from the Poisson distribution in the number of electrons thermally generated within the semiconductor), and the system's inherent readout noise due to the processing of generated charge carriers. Considering these factors, the signalto-noise ratio can be expressed as ${ }^{81}$

$$
\mathrm{S} / \mathrm{N}\left(\delta t_{\text {aq. }}\right)=P Q \delta t_{\text {aq. }} /\left(\left(P Q \delta t_{\text {aq. }}+D \delta t_{\text {aq. }}+R^{2}\right)^{1 / 2}\right)
$$

with $\delta t_{\text {aq. }}$ being the acquisition time, $P$, the photon flux, $Q$ the quantum efficiency of the detector, $D$, the dark current, and $R$, the read out noise. For simplicity, the wavelength dependence of the photon flux and quantum efficiency is neglected here. For a quantitative $\mathrm{S} / \mathrm{N}$ ratio comparison between the WLL/RO and WLL/PRISM schemes, it is important that the photon flux arriving at the detector is comparable for both excitation techniques. As the photon flux stands for photon density over time, it scales with power. As described above, both illumination methodologies use a lens either to focus the WLL at the TIR angle onto the substrate (i.e., WLL/PRISM) or to focus the incoming WLL on the light stop of the reflecting objective (i.e., WLL/RO). To be able to compare the exciting photon fluxes, we measured the power of the WLL using a photodiode detector (Thorlabs, PM100A) with the conversion efficiency set to $\lambda=637 \mathrm{~nm}$ after the respective focusing lens of each illumination scheme. The measured mean power was $P_{637 \mathrm{~nm}}=141 \mathrm{~mW}$ for the WLL/RO configuration and $P_{637 \mathrm{~nm}}=$ $160 \mathrm{~mW}$ for the WLL/PRISM approach. Besides the excitation power, the microscope magnification is a second crucial parameter that influences the amount of detected photons. Overall, a higher magnification decreases the photon flux that arrives from the AuNRs at the camera sensor. Thus, for the S/ $\mathrm{N}$ ratio assessment, the magnification of both systems needs to be similar. Accordingly, the magnification of the reflecting objective $(74 \times)$ was increased by a factor of 1.6 using an internal lens of the microscope. The resulting magnification of $118 \times$ for the WLL/RO method is approximately equal to the magnification of the WLL/PRISM geometry $(125 \times)$. The similar power and similar magnification used for the WLL/RO and WLL/PRISM configurations allowed a comparison of the $\mathrm{S} / \mathrm{N}$ ratio in the scattering spectra of individual AuNRs. 
Our primary goal was to find the shortest possible camera acquisition time $\delta t_{\text {aq. }}$ for a certain $\mathrm{S} / \mathrm{N}$ ratio $\left(\delta t_{\text {aq. }}\right)$ for the two different illumination approaches. As presented in Supporting Information Section 5, Figure S8, the WLL/PRISM configuration could reach $\mathrm{S} / \mathrm{N}$ ratios above 100 within only $1 \mathrm{~ms}$. The $\mathrm{S} / \mathrm{N}$ ratios from the spectra of individual AuNRs were calculated as described in Supporting Information Section 7, Figure S11, and example spectra for the WLL/PRISM and the WLL/RO geometries are given in Figures S12 and S13, respectively. Longer integration times than $1 \mathrm{~ms}$ already led to a local saturation of the CMOS sensor (saturation charge of 30 000 electrons). For the different individual AuNRs measured, we observed a distribution of $\mathrm{S} / \mathrm{N}$ ratios (Figure S8), likely the result of an inhomogeneous size distribution among the AuNRs and potentially a gradient in the spatial evanescent wave excitation (Figure 3b). Nonetheless, the majority of the $\mathrm{S} / \mathrm{N}$ ratios were sufficient for a distinct spectral mapping with an exposure time of only $1 \mathrm{~ms}$. This integration time could be further reduced to reach sub-millisecond time resolution, e.g., $\delta t_{\text {aq. }}=100 \mu \mathrm{s}$. However, the read out time of our CMOS camera sensor is limited by the amount of pixels that are simultaneously active. An acquisition time less than $1 \mathrm{~ms}$ is only possible if the image size is cropped and the amount of detected pixels is reduced, leading to an unwanted decrease in the spatial NP throughput. For the WLL/RO approach, the extracted S/N ratios were below 25 for $1-20 \mathrm{~ms}$ camera acquisition times (Figure S9). For small spherical AuNPs with a diameter of $\sim 35$ $\mathrm{nm}$, we could still detect distinct SHI spectra using the WLL/ PRISM excitation but needed a camera acquisition time of $1 \mathrm{~s}$ (Figure S10).

Finally, we investigated the polarization sensitivity of the WLL/PRISM and WLL/RO illumination schemes. For testing the polarization quality, a mechanical polarizer (Thorlabs) was positioned before the corresponding focusing lenses in the WLL/PRISM and WLL/RO setups. After implementation of the polarizer, the excitation power was again measured behind the focusing lenses. The excitation power dropped $\sim 60 \%$ for both setups in comparison with the $\mathrm{S} / \mathrm{N}$ ratio measurements performed without the polarizer (Figure S8). Consequently, the photon flux that excited the AuNRs decreased for both excitation schemes and we therefore increased the camera acquisition times to $5 \mathrm{~ms}$ for the WLL/PRISM and to $30 \mathrm{~ms}$ for the WLL/RO method to achieve similar scattering intensities. Next, the polarizer was rotated in $45^{\circ}$ steps and the power was measured for each position. The power showed the same weak angle dependency for the two illumination configurations (Supporting Information Section 6, Table S2). We measured the spectral dispersion of individual AuNRs with the CMOS 2 camera and analyzed the integrated intensity for each position of the polarizer for both excitation schemes (Figure 6d,e). We determined the integrated scattering intensity $I$ as

$$
\begin{aligned}
I & =\frac{1}{N}\left(\sum_{i=0}^{N} I_{i, \mathrm{~S} \mathrm{CMOS} 2}\right)-\frac{1}{N}\left(\sum_{i=0}^{N} I_{i, \mathrm{BG} \mathrm{CMOS} 2}\right) \\
& =\overline{I_{\mathrm{S} \mathrm{CMOS} 2}}-\overline{I_{\mathrm{BG} \mathrm{CMOS} 2}}
\end{aligned}
$$

with $N$ being the amount of pixels $i$ that defined the region of interest centering signal $S$ or background BG. Next, we fitted the integrated intensity with the following formula

$$
I=P(1+M \cos (2(\Theta-\Phi)))
$$

Here, $I$ stands for the integrated scattering intensity after background subtraction, $P$ represents a fitting parameter, $M$ is the modulation depth (amplitude), $\Theta$ is the angle of incident light polarization, and $\Phi$ is the AuNR orientation. Because AuNRs behave as dipole scatterers at the longitudinal plasmon resonance, ${ }^{46}$ the modulation depth is a measure of the polarization control. We found that the WLL/PRISM scheme allows good polarization control with values of $M=0.8$ (Figure $6 \mathrm{~d}$ ) for integration times around $5 \mathrm{~ms}$. The polarization control was significantly improved for the WLL/RO configuration with a perfect modulation amplitude of $M=1.0$ (Figure 6e). However, the improved polarization control came at the expense of a longer CMOS camera integration time ( $\sim 30 \mathrm{~ms})$ when ensuring similar photon counts.

\section{CONCLUSIONS}

We have presented a novel two-detector approach to parallel single-particle spectroscopic imaging that allows the in situ and high-quality mapping of $\sim 100$ individual plasmonic NPs. The two-detector approach will in the future also enable spectroscopic imaging of nonstationary NPs and particle growth from small and initially nondetectable seeds. The spectra showed signal-to-noise ratios greater than 100 with an integration time of only $1 \mathrm{~ms}$ while using a high-intensity evanescent wave excitation. We also specified an alternative dark-field excitation approach that utilizes a combination of a supercontinuum laser and a reflecting objective for polarizationcontrolled SHI. As plasmonic NPs provide unique opportunities for photocatalysis, photovoltaics, and electrochemistry, understanding the effect of particle heterogeneity on fast, irreversible plasmon-mediated processes is crucial for their efficient application in a variety of research fields. SHI overcomes the problem with commonly applied spectral analysis methods that observe one particle at a time: ${ }^{82}$ although one NP is monitored, the others are simultaneously subjected to fast irreversible changes that stay uncovered. Our spectroscopic mapping technique allows the detection of the spectral response of many NPs in parallel with fast time resolution, high spectral sensitivity, high $\mathrm{S} / \mathrm{N}$ values, and precise polarization control. We have successfully applied SHI to the heterogeneous gold oxide formation at the surface of AuNRs when subjected to an electrochemical potential.

\section{ASSOCIATED CONTENT}

\section{S Supporting Information}

The Supporting Information is available free of charge on the ACS Publications website at DOI: 10.1021/acs.jpcc.8b01398.

Calibration of the CMOS cameras, characterization of AuNRs, spectra analysis, comparison of AuNR spectra measured by SHI and spectrograph/CCD camera setup, SHI of an AuNR array, measurement of the WLL power, calculation of the signal-to-noise ratio, monitoring the electrochemical redox tuning of the LSPR of single AuNRs, noise level of the SHI setup, and lithography of AuNRs (PDF)

\section{AUTHOR INFORMATION}

\section{Corresponding Authors}

*E-mail: cflandes@rice.edu (C.F.L.).

*E-mail: slink@rice.edu (S.L.).

ORCID $\odot$

Yi-Yu Cai: 0000-0002-2699-9073 
Calum Kinnear: 0000-0002-5663-9933

Wei-Shun Chang: 0000-0002-0251-4449

Paul Mulvaney: 0000-0002-8007-3247

Christy F. Landes: 0000-0003-4163-6497

Stephan Link: 0000-0002-4781-930X

\section{Author Contributions}

"S.R.K. and K.W.S. contributed equally.

\section{Author Contributions}

The manuscript was written through contributions of all authors. All authors have given approval to the final version of the manuscript.

\section{Notes}

The authors declare no competing financial interest.

\section{ACKNOWLEDGMENTS}

The DOE BES (DE-SC0016534 to SL and CFL) supported B.S.H. who performed the electrochemical measurements and W.-S.C. who designed experiments and setup, performed experiments, and wrote the manuscript with direction from C.F.L. and S.L. S. R. Kirchner thanks the German Research Foundation (DFG) for support of this work, while K. W. Smith acknowledges that this material is based upon work supported by the National Science Foundation Graduate Research Fellowship Program (0940902). This work was also partially supported by the Robert A. Welch Foundation (C-1664 to S.L. and C-1787 to C.F.L.), the National Science Foundation (CHE1507745 to S.L.), and the Air Force Office of Scientific Research (MURI FA9550-15-1-0022 to S.L.).

\section{REFERENCES}

(1) Mukherjee, S.; Libisch, F.; Large, N.; Neumann, O.; Brown, L. V.; Cheng, J.; Lassiter, J. B.; Carter, E. A.; Nordlander, P.; Halas, N. J. Hot Electrons Do the Impossible: Plasmon-Induced Dissociation of $\mathrm{H} 2$ on Au. Nano Lett. 2012, 13, 240-247.

(2) Mukherjee, S.; Zhou, L.; Goodman, A. M.; Large, N.; AyalaOrozco, C.; Zhang, Y.; Nordlander, P.; Halas, N. J. Hot-ElectronInduced Dissociation of $\mathrm{H} 2$ on Gold Nanoparticles Supported on Sio2. J. Am. Chem. Soc. 2014, 136, 64-67.

(3) Linic, S.; Aslam, U.; Boerigter, C.; Morabito, M. Photochemical Transformations on Plasmonic Metal Nanoparticles. Nat. Mater. 2015, 14, 567-576.

(4) Ingram, D. B.; Linic, S. Water Splitting on Composite PlasmonicMetal/Semiconductor Photoelectrodes: Evidence for Selective Plasmon-Induced Formation of Charge Carriers near the Semiconductor Surface. J. Am. Chem. Soc. 2011, 133, 5202-5205.

(5) Christopher, P.; Xin, H.; Linic, S. Visible-Light-Enhanced Catalytic Oxidation Reactions on Plasmonic Silver Nanostructures. Nat. Chem. 2011, 3, 467-472.

(6) Park, J. Y.; Baker, L. R.; Somorjai, G. A. Role of Hot Electrons and Metal-Oxide Interfaces in Surface Chemistry and Catalytic Reactions. Chem. Rev. 2015, 115, 2781-2817.

(7) Lin, Z.; Wang, X.; Liu, J.; Tian, Z.; Dai, L.; He, B.; Han, C.; Wu, Y.; Zeng, Z.; Hu, Z. On the Role of Localized Surface Plasmon Resonance in Uv-Vis Light Irradiated $\mathrm{Au} / \mathrm{Tio} 2$ Photocatalysis Systems: Pros and Cons. Nanoscale 2015, 7, 4114-4123.

(8) Jing, C.; Rawson, F. J.; Zhou, H.; Shi, X.; Li, W.-H.; Li, D.-W.; Long, Y.-T. New Insights into Electrocatalysis Based on Plasmon Resonance for the Real-Time Monitoring of Catalytic Events on Single Gold Nanorods. Anal. Chem. 2014, 86, 5513-5518.

(9) Thomann, I.; Pinaud, B. A.; Chen, Z.; Clemens, B. M.; Jaramillo, T. F.; Brongersma, M. L. Plasmon Enhanced Solar-to-Fuel Energy Conversion. Nano Lett. 2011, 11, 3440-3446.

(10) Linic, S.; Christopher, P.; Ingram, D. B. Plasmonic-Metal Nanostructures for Efficient Conversion of Solar to Chemical Energy. Nat. Mater. 2011, 10, 911-921.
(11) Zhong, Y.; Ueno, K.; Mori, Y.; Oshikiri, T.; Misawa, H. Cocatalyst Effects on Hydrogen Evolution in a Plasmon-Induced Water-Splitting System. J. Phys. Chem. C 2015, 119, 8889-8897.

(12) Oshikiri, T.; Ueno, K.; Misawa, H. Selective Dinitrogen Conversion to Ammonia Using Water and Visible Light through Plasmon-Induced Charge Separation. Angew. Chem. 2016, 4010-4014.

(13) Narang, P.; Sundararaman, R.; Atwater, H. A. Plasmonic Hot Carrier Dynamics in Solid-State and Chemical Systems for Energy Conversion. Nanophotonics 2016, 5, 96.

(14) Atwater, H. A.; Polman, A. Plasmonics for Improved Photovoltaic Devices. Nat. Mater. 2010, 9, 205-213.

(15) Wu, K.; Chen, J.; McBride, J.; Lian, T. Efficient Hot-Electron Transfer by a Plasmon-Induced Interfacial Charge-Transfer Transition. Science 2015, 349, 632-635.

(16) Carattino, A.; Khatua, S.; Orrit, M. In Situ Tuning of Gold Nanorod Plasmon through Oxidative Cyanide Etching. Phys. Chem. Chem. Phys. 2016, 18, 15619-15624.

(17) Ament, I.; Prasad, J.; Henkel, A.; Schmachtel, S.; Sönnichsen, C. Single Unlabeled Protein Detection on Individual Plasmonic Nanoparticles. Nano Lett. 2012, 12, 1092-1095.

(18) Rosman, C.; Prasad, J.; Neiser, A.; Henkel, A.; Edgar, J.; Sönnichsen, C. Multiplexed Plasmon Sensor for Rapid Label-Free Analyte Detection. Nano Lett. 2013, 13, 3243-3247.

(19) Ahijado-Guzmán, R.; Prasad, J.; Rosman, C.; Henkel, A.; Tome, L.; Schneider, D.; Rivas, G.; Sönnichsen, C. Plasmonic Nanosensors for Simultaneous Quantification of Multiple Protein-Protein Binding Affinities. Nano Lett. 2014, 14, 5528-5532.

(20) Zhai, Y.; DuChene, J. S.; Wang, Y.-C.; Qiu, J.; Johnston-Peck, A. C.; You, B.; Guo, W.; DiCiaccio, B.; Qian, K.; Zhao, E. W.; et al. Polyvinylpyrrolidone-Induced Anisotropic Growth of Gold Nanoprisms in Plasmon-Driven Synthesis. Nat. Mater. 2016, 15, 889-895.

(21) Langille, M. R.; Zhang, J.; Personick, M. L.; Li, S.; Mirkin, C. A. Stepwise Evolution of Spherical Seeds into 20-Fold Twinned Icosahedra. Science 2012, 337, 954-957.

(22) Zhang, J.; Langille, M. R.; Mirkin, C. A. Synthesis of Silver Nanorods by Low Energy Excitation of Spherical Plasmonic Seeds. Nano Lett. 2011, 11, 2495-2498.

(23) Zhang, J.; Li, S.; Wu, J.; Schatz, G. C.; Mirkin, C. A. PlasmonMediated Synthesis of Silver Triangular Bipyramids. Angew. Chem., Int. Ed. 2009, 48, 7787-7791.

(24) Langille, M. R.; Zhang, J.; Mirkin, C. A. Plasmon-Mediated Synthesis of Heterometallic Nanorods and Icosahedra. Angew. Chem., Int. Ed. 2011, 50, 3543-3547.

(25) Jin, R.; Cao, Y.; Mirkin, C. A.; Kelly, K.; Schatz, G. C.; Zheng, J. Photoinduced Conversion of Silver Nanospheres to Nanoprisms. Science 2001, 294, 1901-1903.

(26) Jin, R.; Cao, Y. C.; Hao, E.; Métraux, G. S.; Schatz, G. C.; Mirkin, C. A. Controlling Anisotropic Nanoparticle Growth through Plasmon Excitation. Nature 2003, 425, 487-490.

(27) Langille, M. R.; Personick, M. L.; Mirkin, C. A. PlasmonMediated Syntheses of Metallic Nanostructures. Angew. Chem., Int. Ed. 2013, 52, 13910-13940.

(28) Violi, I. L.; Gargiulo, J.; von Bilderling, C.; Cortés, E.; Stefani, F. D. Light-Induced Polarization-Directed Growth of Optically Printed Gold Nanoparticles. Nano Lett. 2016, 16, 6529-6533.

(29) Simoncelli, S.; Roller, E.-M.; Urban, P.; Schreiber, R.; Turberfield, A. J.; Liedl, T.; Lohmüller, T. Quantitative SingleMolecule Surface-Enhanced Raman Scattering by Optothermal Tuning of DNA Origami-Assembled Plasmonic Nanoantennas. ACS Nano 2016, 10, 9809-9815.

(30) Zaleski, S.; Cardinal, M. F.; Klingsporn, J. M.; Van Duyne, R. P. Observing Single, Heterogeneous, One-Electron Transfer Reactions. J. Phys. Chem. C 2015, 119, 28226-28234.

(31) Shegai, T.; Vaskevich, A.; Rubinstein, I.; Haran, G. Raman Spectroelectrochemistry of Molecules within Individual Electromagnetic Hot Spots. J. Am. Chem. Soc. 2009, 131, 14390-14398.

(32) Li, C. Y.; Dong, J. C.; Jin, X.; Chen, S.; Panneerselvam, R.; Rudnev, A. V.; Yang, Z. L.; Li, J. F.; Wandlowski, T.; Tian, Z. Q. In Situ Monitoring of Electrooxidation Processes at Gold Single Crystal 
Surfaces Using Shell-Isolated Nanoparticle-Enhanced Raman Spectroscopy. J. Am. Chem. Soc. 2015, 137, 7648-7651.

(33) Zhao, Y.; Saleh, A. A.; Dionne, J. A. Enantioselective Optical Trapping of Chiral Nanoparticles with Plasmonic Tweezers. ACS Photonics 2016, 3, 304-309.

(34) Zhang, W.; Caldarola, M.; Pradhan, B.; Orrit, M. Gold Nanorod-Enhanced Fluorescence Enables Single-Molecule Electrochemistry of Methylene Blue. Angew. Chem., Int. Ed. 2017, 3620-3623.

(35) Novo, C.; Funston, A. M.; Mulvaney, P. Direct Observation of Chemical Reactions on Single Gold Nanocrystals Using Surface Plasmon Spectroscopy. Nat. Nanotechnol. 2008, 3, 598-602.

(36) Novo, C.; Funston, A. M.; Gooding, A. K.; Mulvaney, P. Electrochemical Charging of Single Gold Nanorods. J. Am. Chem. Soc. 2009, 131, 14664-14666.

(37) Byers, C. P.; Zhang, H.; Swearer, D. F.; Yorulmaz, M.; Hoener, B. S.; Huang, D.; Hoggard, A.; Chang, W.-S.; Mulvaney, P.; Ringe, E.; et al. From Tunable Core-Shell Nanoparticles to Plasmonic Drawbridges: Active Control of Nanoparticle Optical Properties. Sci. Adv. 2015, 1, No. e1500988.

(38) Leroux, Y.; Lacroix, J. C.; Fave, C.; Trippe, G.; Félidj, N.; Aubard, J.; Hohenau, A.; Krenn, J. R. Tunable Electrochemical Switch of the Optical Properties of Metallic Nanoparticles. ACS Nano 2008, 2, $728-732$.

(39) Hoener, B. S.; Byers, C. P.; Heiderscheit, T. S.; De Silva Indrasekara, A. S.; Hoggard, A.; Chang, W.-S.; Link, S.; Landes, C. F. Spectroelectrochemistry of Halide Anion Adsorption and Dissolution of Single Gold Nanorods. J. Phys. Chem. C 2016, 20604-20612.

(40) Byers, C. P.; Hoener, B. S.; Chang, W.-S.; Yorulmaz, M.; Link, S.; Landes, C. F. Single-Particle Spectroscopy Reveals Heterogeneity in Electrochemical Tuning of the Localized Surface Plasmon. J. Phys. Chem. B 2014, 118, 14047-14055.

(41) Chirea, M.; Collins, S. S.; Wei, X.; Mulvaney, P. Spectroelectrochemistry of Silver Deposition on Single Gold Nanocrystals. J. Phys. Chem. Lett. 2014, 5, 4331-4335.

(42) Li, Z.; Mao, W.; Devadas, M. S.; Hartland, G. V. Absorption Spectroscopy of Single Optically Trapped Gold Nanorods. Nano Lett. 2015, 15, 7731-7735.

(43) Hu, M.; Chen, J.; Li, Z.-Y.; Au, L.; Hartland, G. V.; Li, X.; Marquez, M.; Xia, Y. Gold Nanostructures: Engineering Their Plasmonic Properties for Biomedical Applications. Chem. Soc. Rev. 2006, 35, 1084-1094.

(44) Huergo, M. A.; Maier, C. M.; Castez, M. F.; Vericat, C.; Nedev, S.; Salvarezza, R. C.; Urban, A. S.; Feldmann, J. Optical Nanoparticle Sorting Elucidates Synthesis of Plasmonic Nanotriangles. ACS Nano 2016, 10, 3614-3621.

(45) Link, S.; Mohamed, M.; El-Sayed, M. Simulation of the Optical Absorption Spectra of Gold Nanorods as a Function of Their Aspect Ratio and the Effect of the Medium Dielectric Constant. J. Phys. Chem. B 1999, 103, 3073-3077.

(46) Link, S.; El-Sayed, M. A. Spectral Properties and Relaxation Dynamics of Surface Plasmon Electronic Oscillations in Gold and Silver Nanodots and Nanorods. J. Phys. Chem. B 1999, 103, 84108426.

(47) Sönnichsen, C.; Franzl, T.; Wilk, T.; von Plessen, G.; Feldmann, J.; Wilson, O.; Mulvaney, P. Drastic Reduction of Plasmon Damping in Gold Nanorods. Phys. Rev. Lett. 2002, 88, No. 077402.

(48) Brown, A. M.; Sundararaman, R.; Narang, P.; Goddard, W. A., III; Atwater, H. A. Nonradiative Plasmon Decay and Hot Carrier Dynamics: Effects of Phonons, Surfaces, and Geometry. ACS Nano 2016, 10, 957-966.

(49) Scholl, J. A.; Garcia-Etxarri, A.; Aguirregabiria, G.; Esteban, R.; Narayan, T. C.; Koh, A. L.; Aizpurua, J.; Dionne, J. A. Evolution of Plasmonic Metamolecule Modes in the Quantum Tunneling Regime. ACS Nano 2016, 10, 1346-1354.

(50) Sun, L.; Ma, T.; Yang, S.-C.; Kim, D.-K.; Lee, G.; Shi, J.; Martinez, I.; Yi, G.-R.; Shvets, G.; Li, X. Interplay between Optical Bianisotropy and Magnetism in Plasmonic Metamolecules. Nano Lett. 2016, 16, 4322-4328.
(51) Wu, J.; et al. Broadband Efficiency Enhancement in Quantum Dot Solar Cells Coupled with Multispiked Plasmonic Nanostars. Nano Energy 2015, 13, 827-835.

(52) Trügler, A.; Tinguely, J.-C.; Krenn, J. R.; Hohenau, A.; Hohenester, U. Influence of Surface Roughness on the Optical Properties of Plasmonic Nanoparticles. Phys. Rev. B 2011, 83, No. 081412.

(53) Pecharromán, C.; Pérez-Juste, J.; Mata-Osoro, G.; Liz-Marzán, L. M.; Mulvaney, P. Redshift of Surface Plasmon Modes of Small Gold Rods Due to Their Atomic Roughness and End-Cap Geometry. Phys. Rev. B 2008, 77, No. 035418.

(54) Juluri, B. K.; Zheng, Y. B.; Ahmed, D.; Jensen, L.; Huang, T. J. Effects of Geometry and Composition on Charge-Induced Plasmonic Shifts in Gold Nanoparticles. J. Phys. Chem. C 2008, 112, 7309-7317.

(55) Lee, K.-S.; El-Sayed, M. A. Gold and Silver Nanoparticles in Sensing and Imaging: Sensitivity of Plasmon Response to Size, Shape, and Metal Composition. J. Phys. Chem. B 2006, 110, 19220-19225.

(56) Wu, Y.; et al. Intrinsic Optical Properties and Enhanced Plasmonic Response of Epitaxial Silver. Adv. Mater. 2014, 26, 61066110.

(57) Chen, M.; Shao, L.; Kershaw, S. V.; Yu, H.; Wang, J.; Rogach, A. L.; Zhao, N. Photocurrent Enhancement of Hgte Quantum Dot Photodiodes by Plasmonic Gold Nanorod Structures. ACS Nano 2014, 8, 8208-8216.

(58) Sheldon, M. T.; Van de Groep, J.; Brown, A. M.; Polman, A.; Atwater, H. A. Plasmoelectric Potentials in Metal Nanostructures. Science 2014, 346, 828-831.

(59) Smith, K. W.; Zhao, H.; Zhang, H.; Sánchez-Iglesias, A.; Grzelczak, M.; Wang, Y. M.; Chang, W.-S.; Nordlander, P.; LizMarzan, L. M.; Link, S. Chiral and Achiral Nanodumbbell Dimers: The Effect of Geometry on Plasmonic Properties. ACS Nano 2016, 61806188.

(60) Olson, J.; Manjavacas, A.; Basu, T.; Huang, D.; Schlather, A. E.; Zheng, B.; Halas, N. J.; Nordlander, P.; Link, S. High Chromaticity Aluminum Plasmonic Pixels for Active Liquid Crystal Displays. ACS Nano 2016, 10, 1108-1117.

(61) Don, H.; Johnson, R. U. Statistical Signal Processing; IEEE, 2017.

(62) Gonzalez, R. C.; Woods, R. E. Digital Image Processing; Prentice Hall, 2008.

(63) Stathaki, T. Image Fusion: Algorithms and Applications; Academic Press, 2008.

(64) Wulf, V.; Knoch, F.; Speck, T.; Sönnichsen, C. Gold Nanorods as Plasmonic Sensors for Particle Diffusion. J. Phys. Chem. Lett. 2016, 7, 4951-4955.

(65) Bingham, J. M.; Willets, K. A.; Shah, N. C.; Andrews, D. Q.; Van Duyne, R. P. Localized Surface Plasmon Resonance Imaging: Simultaneous Single Nanoparticle Spectroscopy and Diffusional Dynamics. J. Phys. Chem. C 2009, 113, 16839-16842.

(66) El-Khoury, P. Z.; Joly, A. G.; Hess, W. P. Hyperspectral Dark Field Optical Microscopy of Single Silver Nanospheres. J. Phys. Chem. C 2016, 120, 7295-7298.

(67) Cheng, J.; Liu, Y.; Cheng, X.; He, Y.; Yeung, E. S. Real Time Observation of Chemical Reactions of Individual Metal Nanoparticles with High-Throughput Single Molecule Spectral Microscopy. Anal. Chem. 2010, 82, 8744-8749.

(68) Ma, Y.; Shortreed, M. R.; Yeung, E. S. High-Throughput SingleMolecule Spectroscopy in Free Solution. Anal. Chem. 2000, 72, 46404645.

(69) Hao, J.; Xiong, B.; Cheng, X.; He, Y.; Yeung, E. S. HighThroughput Sulfide Sensing with Colorimetric Analysis of Single AuAg Core-Shell Nanoparticles. Anal. Chem. 2014, 86, 4663-4667.

(70) Nusz, G. J.; Marinakos, S. M.; Rangarajan, S.; Chilkoti, A. DualOrder Snapshot Spectral Imaging of Plasmonic Nanoparticles. Appl. Opt. 2011, 50, 4198-4206.

(71) Xu, D.; He, Y.; Yeung, E. S. Direct Observation of the Orientation Dynamics of Single Protein-Coated Nanoparticles at Liquid/Solid Interfaces. Angew. Chem., Int. Ed. 2014, 53, 6951-6955.

(72) Xiong, B.; Zhou, R.; Hao, J.; Jia, Y.; He, Y.; Yeung, E. S. Highly Sensitive Sulphide Mapping in Live Cells by Kinetic Spectral Analysis 
of Single Au-Ag Core-Shell Nanoparticles. Nat. Commun. 2013, 4, No. 1708.

(73) Shi, X.; Xie, Z.; Song, Y.; Tan, Y.; Yeung, E. S.; Gai, H. Superlocalization Spectral Imaging Microscopy of a Multicolor Quantum Dot Complex. Anal. Chem. 2012, 84, 1504-1509.

(74) Becker, J.; Schubert, O.; Sönnichsen, C. Gold Nanoparticle Growth Monitored in Situ Using a Novel Fast Optical Single-Particle Spectroscopy Method. Nano Lett. 2007, 7, 1664-1669.

(75) Zheng, Z.; Tachikawa, T.; Majima, T. Plasmon-Induced Spatial Electron Transfer between Single Au Nanorods and Ald-Coated Tio 2: Dependence on Tio 2 Thickness. Chem. Commun. 2015, 51, 1437314376.

(76) Hoener, B. S.; Zhang, H.; Heiderscheit, T. S.; Kirchner, S. R.; De Silva Indrasekara, A. S.; Baiyasi, R.; Cai, Y.-Y.; Nordlander, P.; Link, S.; Landes, C. F.; Chang, W.-S. Spectral Response of Plasmonic Gold Nanoparticles to Capacitive Charging: Morphology Effects. J. Phys. Chem. Lett. 2017, 8, 2681-2688.

(77) Wang, Y.; Shan, X.; Wang, H.; Wang, S.; Tao, N. Plasmonic Imaging of Surface Electrochemical Reactions of Single Gold Nanowires. J. Am. Chem. Soc. 2017, 139, 1376-1379.

(78) Li, C.-Y.; Dong, J.-C.; Jin, X.; Chen, S.; Panneerselvam, R.; Rudnev, A. V.; Yang, Z.-L.; Li, J.-F.; Wandlowski, T.; Tian, Z.-Q. In Situ Monitoring of Electrooxidation Processes at Gold Single Crystal Surfaces Using Shell-Isolated Nanoparticle-Enhanced Raman Spectroscopy. J. Am. Chem. Soc. 2015, 137, 7648-7651.

(79) Chen, A.; Lipkowski, J. Electrochemical and Spectroscopic Studies of Hydroxide Adsorption at the Au (111) Electrode. J. Phys. Chem. B 1999, 103, 682-691.

(80) Axelrod, D. Total Internal Reflection Fluorescence Microscopy in Cell Biology. Traffic 2001, 2, 764-774.

(81) Fellers, T. J.; Vogt, K. M.; Davidson, M. W. Tutorials - CCD Signal-to-Noise Ratio; Nikon Microscopy-The Source for Microscopy Education.

(82) Collins, S. S.; Wei, X.; McKenzie, T. G.; Funston, A. M.; Mulvaney, P. Single Gold Nanorod Charge Modulation in an Ion Gel Device. Nano Lett. 2016, 16, 6863-6869. 